\title{
ERROR ANALYSIS OF MOTION CORRECTION METHOD FOR LASER SCANNING OF MOVING OBJECTS
}

\author{
Salil Goel and Bharat Lohani \\ Geoinformatics Laboratory \\ Department of Civil Engineering \\ Indian Institute of Technology Kanpur \\ Kanpur, Uttar Pradesh-208016, India \\ goel.salilg@gmail.com, blohani@iitk.ac.in
}

Commission V

KEY WORDS: Error Modelling, Motion Correction, GPS/INS Integration, Laser Scanning

\begin{abstract}
:
The limitation of conventional laser scanning methods is that the objects being scanned should be static. The need of scanning moving objects has resulted in the development of new methods capable of generating correct 3D geometry of moving objects. Limited literature is available showing development of very few methods capable of catering to the problem of object motion during scanning. All the existing methods utilize their own models or sensors. Any studies on error modelling or analysis of any of the motion correction methods are found to be lacking in literature. In this paper, we develop the error budget and present the analysis of one such 'motion correction' method. This method assumes availability of position and orientation information of the moving object which in general can be obtained by installing a POS system on board or by use of some tracking devices. It then uses this information along with laser scanner data to apply correction to laser data, thus resulting in correct geometry despite the object being mobile during scanning. The major application of this method lie in the shipping industry to scan ships either moving or parked in the sea and to scan other objects like hot air balloons or aerostats. It is to be noted that the other methods of 'motion correction' explained in literature can not be applied to scan the objects mentioned here making the chosen method quite unique. This paper presents some interesting insights in to the functioning of 'motion correction' method as well as a detailed account of the behavior and variation of the error due to different sensor components alone and in combination with each other. The analysis can be used to obtain insights in to optimal utilization of available components for achieving the best results.
\end{abstract}

\section{INTRODUCTION}

Conventional laser scanning methods suffer from the limitation that the objects being scanned should be static. Laser scanning of moving objects result in random point cloud data which do not represent the correct geometry of the object. For example, a ship parked in sea would not appear in its true form in the collected data. This need of scanning moving objects has resulted in to development of new methods like the method proposed by Blais et al.(2004) which first creates a rough, partial and distorted estimate of the model and then refines on it by using new range information using the ICP based algorithm. The other algorithm proposed by Weise et al.(2007) works by combining stereo and active illumination and uses a closed form expression for motion error compensation. The latest method for motion correction is proposed by Goel and Lohani(2014) which combines a laser scanner with position and attitude measurement devices to first compute the six degrees of motion and then use the same to compensate for motion error. The three methods propose their own unique ways of dealing with the problem of scanning of moving objects. Of the three available methods, the algorithms proposed by Blais et al.(2004) and Weise et al.(2007) can only be applied for objects located to short distance from the laser scanner.

Literature shows no attempt has been made on error analysis, modeling, budgeting or propagation of any of the proposed methods. This paper attempts to study the nature of errors, develop an error budget and study the effect of various sensors on the overall performance for one of the motion correction methods: the method proposed by Goel and Lohani (2014). As of now, this paper presents the analysis for the case of a static 3D laser scanner and moving object, though the method (Goel and Lohani (2014)) is well capable of handling the cases of moving laser scanner. Even though the study can be presented for any combination of sensors, 3D laser scanner from Optech and POSLV 220 GPS/INS system from Applanix are used in the simulation study. Though this method can work even in the absence of POS if position and orientation information are derived using alternative means, the analysis is presented considering the case of Applanix POS system. Since POS is an important component of this method, the effect of using different POS systems is also presented. The major application of this method lie in the shipping industry to scan ships either moving or parked in the sea and to scan other objects like hot air balloons or aerostats where the other two methods would fail to generate any results. This paper assumes presence of no systematic errors in the system and takes in to account the contribution of random error sources only as well as ignores any time synchronization erros. Also, the effect of material properties and incidence angle are not modelled in this paper. Through this analysis, general error trends can be derived which are applicable to any combination of the sensors and can be used by other researchers.

The paper is divided in to five sections. Section 2 explains the motion correction algorithm by Goel and Lohani (2014) in brief. Section 3 develops the error model and explains the same in detail. Section 4 presents and analyses the results of the simulation. Conclusions and discussions are given in section 5 .

\section{MOTION CORRECTION METHOD}

The motion correction algorithm proposed by Goel and Lohani (2014) generates true geometry of a moving object which is be- 
ing scanned by a standard laser scanner. It assumes that position and orientation information of the moving object can be obtained either remotely using tracking devices or by the use of positioning and attitude sensors or by some other means. The crux of this method lies in the transformation of the recorded laser scanner data (which is generally in a local laser scanner coordinate system) to a new coordinate system called Object Body Coordinate System (OBCS), which is fixed with the object and is rigid with respect to it via a global coordinate system. The transformation from global coordinate system to OBCS is depicted in figure 1. After making some suitable assumptions, the motion

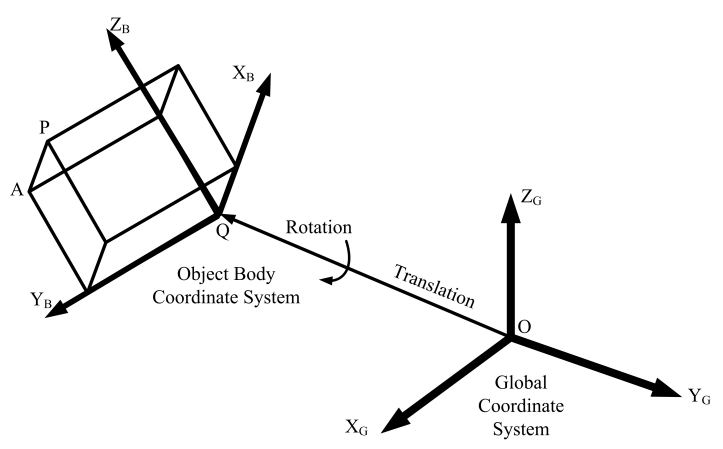

Figure 1: Transformation from Global to OBCS. $X_{G} Y_{G} Z_{G}$ represents Global coordinate system and $X_{B} Y_{B} Z_{B}$ represents OBCS. Q is the origin of OBCS, $\mathrm{P}$ is the point where GPS/INS assembly is mounted and $\mathrm{A}$ is any point located on the object (i.e. cuboid). (After Goel and Lohani (2004)).

correction equation (as proposed by Goel and Lohani (2014)) can be summed up as follows.

$$
r^{B}=\left({ }_{E} R_{t}^{I}\right)\left({ }_{G} R_{t}^{E}\right)\left(r_{t}^{G}-P_{t}^{G}\right)
$$

Where ${ }_{E} R_{t}^{I}$ and ${ }_{G} R_{t}^{E}$ denote $(3 \times 3)$ rotation matrices from Earth Tangential to IMU body and Global to Earth Tangential coordinate systems, respectively at a given time $t$. The notations $r_{t}^{G}$ and $r^{B}$ denote the coordinates of a point on the moving object in Global coordinate system at time $t$ and in OBCS, respectively. $P_{t}^{G}$ denotes the coordinates of the point at which GPS/INS system is rigidly installed on the object. The coordinates given by $r^{B}$ represent the 'motion-corrected coordinates' and depict the true geometry of the moving object.

\section{ERROR MODEL}

The motion correction error model should take in to account the contribution of all random error sources. These sources include noise due to instruments used i.e., laser scanner, GPS, INS and due to the adopted orientation method. A $(3 \times 3)$ covariance matrix is given by the error model using which the maximum propagated error at any level of confidence can be computed. Systematic errors are not being considered here, as the purpose of this paper is to look into the effect of only random errors in observed quantities. The covariance matrix $C_{X}$ is computed using the law of propagation of variances as,

$$
C_{X}=J C_{X}^{o b s} J^{T}
$$

where $J$ is the Jacobian and the matrix $C_{X}^{o b s}$ is composed of the uncertainties in the following observed quantities.

- Angle, Angle and Range (for static 3D laser scanner) given by the laser scanner.
- Scanner orientation parameters for laser scanner: 3 Angles and 3 Translation elements.

- Latitude, Longitude, Height provided by the GPS installed on the moving object.

- Roll, Pitch, Yaw provided by the INS (or IMU) system installed on-board the moving object.

The observation covariance matrix $C_{X}^{o b s}$ is constructed using the above mentioned uncertainties with diagonal elements as standard errors in each of the observations, thus making the size of the covariance matrix be $(15 \times 15)$. The size of Jacobian is $(3 \times 15)$ thus resulting in the computed covariance matrix $C_{X}$ to be of size $(3 \times 3)$. The observation covariance matrix is governed by the components used in the motion correction procedure which are explained below.

\subsection{Optech Laser Scanner}

The measurements observed by the 3D laser scanner are horizontal angle $(\alpha)$, vertical angle $(\beta)$ and laser range $(\rho)$. The coordinates of any point can then be computed from the observed quantities using the following mathematical expression.

$$
r_{t}^{L}=\left[\begin{array}{c}
\rho \cos \alpha \cos \beta \\
\rho \sin \alpha \cos \beta \\
\rho \sin \beta
\end{array}\right]
$$

Where $r_{t}^{L}$ denotes the coordinates of a point in laser scanner coordinate system (denoted by superscript $L$ ) at time $t$. The specifications of Optech ILRIS 3D laser scanner are given in table 1. The error in angular measurement is governed by raw angle mea-

Table 1: Optech ILRIS 3D specifications

\begin{tabular}{|l|c|}
\hline Raw range accuracy ${ }^{\mathrm{a}}$ & $7 \mathrm{~mm}$ \\
\hline Beam divergence & $0.15 \mathrm{mrad}$ \\
\hline Angular accuracy & $80 \mathrm{urad}$ \\
\hline Field of view & $360 \mathrm{deg}$ \\
\hline Maximum range at $80 \%$ reflectivity & $1250 \mathrm{~m}$ \\
\hline Peak Pulse Repetition Frequency (PRF) & $10,000 \mathrm{~Hz}$ \\
\hline \multicolumn{2}{|c|}{${ }^{\mathrm{a}}$ Insignificant change in range error with change in range }
\end{tabular}

surement and footprint size of laser beam. The footprint size is generally specified as the sum of beam diameter at the exit and the expansion in beam diameter due to divergence. Due to finite size of the footprint, the actual point observed by the laser scanner can lie anywhere inside the footprint resulting in an uncertainty in angular location of the point. Hence, the associated angular uncertainty is taken to be the resultant of raw angular accuracy and one-fourth of effective beam diameter (Lichti and Gordon (2004)). The range accuracy is taken to be $7 \mathrm{~mm}$ considering insignificant change in this with laser range.

\subsection{Applanix Position and Orientation System (POS)}

This paper considers Applanix POSLV 220 for the simulation, even though the same analysis can be performed for any of the available POS systems. POS is crucial for accurate determination of 6 degrees of freedom of the moving object. The error sources contributing to the POS arise from GPS and INS observations. Some of the factors which contribute to GPS errors are: (i) Location of satellites with respect to GPS antenna, (ii) Ionospheric and Tropospheric effects, (iii) Satellite and receiver clock errors, (iv) Effect of multipath, (v) Manufacturing defects. INS, composed of gyroscopes and accelerometers, provides the orientation parameters (roll, pitch and yaw) as the end product. It 
also aids GPS in situations of 'loss of lock' which happens especially in urban environments. Each of the components, GPS and INS have their own errors and noise sources which are not modeled individually in this paper, rather information about the overall performance of the integrated system is used to study the effect of this system on overall system performance. The specifications of Applanix POSLV 220 as reported by the manufacturer are given in table 2 . The specifications of other models of the same equipment are also listed in the same table, for comparison.

Table 2: Specifications of POS systems offered by Applanix

\begin{tabular}{|l|l|l|l|}
\hline Parameter & $\begin{array}{l}\text { POSLV } \\
\mathbf{2 2 0}\end{array}$ & $\begin{array}{l}\text { POSLV } \\
\mathbf{4 2 0}\end{array}$ & $\begin{array}{l}\text { POSLV } \\
\mathbf{6 1 0}\end{array}$ \\
\hline $\begin{array}{l}\text { X,Y position } \\
(\mathrm{m})\end{array}$ & 0.02 & 0.02 & 0.02 \\
\hline Z position (m) & 0.05 & 0.05 & 0.05 \\
\hline $\begin{array}{l}\text { Roll and pitch } \\
\text { (deg) }\end{array}$ & 0.02 & 0.015 & 0.005 \\
\hline $\begin{array}{l}\text { True heading } \\
\text { (deg) }\end{array}$ & 0.025 & 0.02 & 0.015 \\
\hline
\end{tabular}

${ }^{\mathrm{B}}$ Standard error $(1 \sigma)$ obtained by various systems in PP mode

\subsection{Laser Scanner orientation parameters}

The POS system generally provides the solution in a Global coordinate system while the data captured by laser scanner is in a local Laser coordinate system. To ensure the compatibility between different coordinate systems, a conversion from the Laser coordinate system to Global coordinate system is needed which is maintained by orientation parameters. In other words, the role of scanner orientation parameters is to transform the data captured by laser scanner in Laser coordinate system to Global coordinate system via a rotation (governed by three angles) and a translation. This transformation from local laser scanner coordinate system to global coordinate system can be expressed by the following equation.

$$
r^{G}={ }_{L} R^{G}\left(r^{L}\right)+T^{G}
$$

Where ${ }_{L} R^{G}$ is the rotation matrix and $T^{G}$ is the translation vector. The most general procedure of determining the rotation matrix and translation vector involves establishing point correspondences between the points in Global and Local coordinate systems and using equation 4 to solve for the rotation and translation elements using the method of least squares adjustment. The error in computed orientation parameters is governed by inaccuracies of the instruments used, adopted surveying procedure and random errors. These individual effects are not modeled in this paper, rather their combined effect on system performance is studied. The standard error assumed in orientation parameters are given in table 3 , which are based on their possible values if realized in field. Two sets of values of orientation parameters are used in

Table 3: Assumed errors in computed orientation parameters

\begin{tabular}{|l|l|l|}
\hline Parameter & Error & (Degraded) Error \\
\hline Roll (deg) & 0.001 & 0.02 \\
\hline Pitch (deg) & 0.001 & 0.02 \\
\hline Yaw (deg) & 0.001 & 0.02 \\
\hline Translation along X (m) & 0.001 & 0.02 \\
\hline Translation along Y (m) & 0.001 & 0.02 \\
\hline Translation along Z (m) & 0.001 & 0.02 \\
\hline
\end{tabular}

the simulation to study their effect, one of which is the optimistic and the other is a degraded set of values. The effect of both these values will be shown in the following section.

\section{RESULTS AND DISCUSSION}

The results section is divided in to two subsections with the first subsection presenting the results of a preliminary simulation where in the motion correction algorithm is applied on a simulated cube and effect of errors is analyzed qualitatively. The second section presents a quantitative and qualitative analysis of the effect of the various sensor components on system performance and verifies and discusses these results with the one obtained in previous subsection.

\subsection{Preliminary Simulation}

A cuboid of size $200 \mathrm{~m} \times 200 \mathrm{~m} \times 100 \mathrm{~m}$ as shown in figure 2 is used for performing the simulation of motion correction algorithm. The POS system is installed on the cuboid at point ' $\mathrm{P}$ ', at the center of the edge $\mathrm{FH}$. The cuboid is moving at rates of $5 \mathrm{~m} / \mathrm{s}$ along $\mathrm{X}$ direction, $1 \mathrm{~m} / \mathrm{s}$ along $\mathrm{Y}$ direction and $0.1 \mathrm{~m} / \mathrm{s}$ along $\mathrm{Z}$ direction. As the object is moving it is undergoing random rotations along the three directions, akin to a car moving on a bumpy road. Due to the object (i.e. cuboid) not being static, the data captured by the laser scanner is erroneous and does not represent the true geometry. To simulate the laser scanner data, the points are chosen randomly on the moving object. These coordinates of the points on the moving object are converted in global coordinate system. Meanwhile, the position and orientation information of the moving cuboid is recorded by the POS installed at point ' $\mathrm{P}$ '. The data captured by the laser scanner when visualized in a visualization software appears as shown in figure 3 . As seen in the

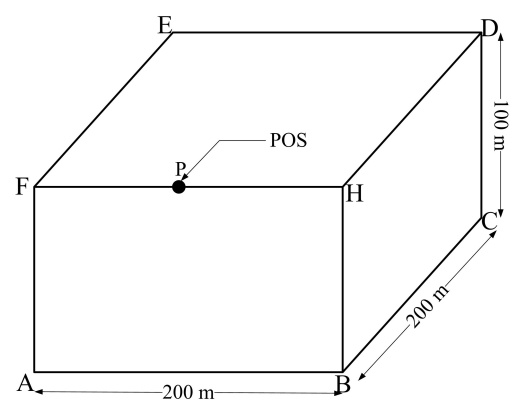

Figure 2: The cuboid used for simulation of motion correction experiment. The POS system is installed on the cuboid at point ' $\mathrm{P}$ '. The cuboid undergoes rotation about its three axis as it is traversing in space.

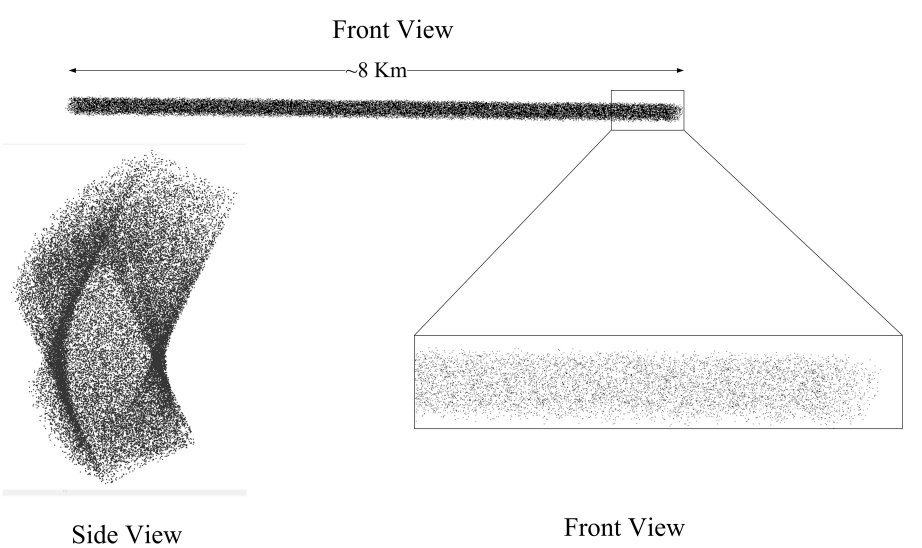

Figure 3: Data corresponding to moving object captured by laser scanner when visualized in a visualization software. The zoomed in views of the front view and side view are shown.

front view in figure 3 , all the data points appear scattered through 
out the region traversed by the moving object, which is about 8 $\mathrm{km}$ in length. The points also seem to follow a rotation pattern as visible in the side view in figure 3. Of course, the appearance of the 'distorted' data is dependent on the trajectory followed by the moving object, scanning pattern and data capturing rate of the laser scanner. The effect of scanning pattern and data capturing rate is not modeled in this paper as it does not add any value to this study. As is clearly visible, figure 3 does not depict the true geometry and thus 'motion correction' algorithm needs to be applied to generate the true geometry. Figure 4 shows the geometry recovered after applying motion correction algorithm. The recov-

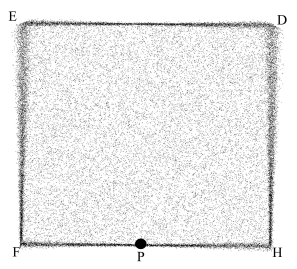

Top view

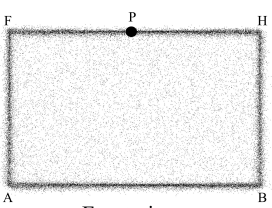

Front view

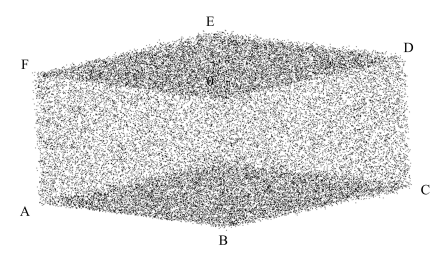

3D view

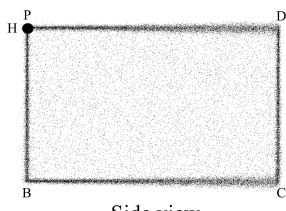

Side view
Figure 4: Geometry recovered after applying motion correction algorithm. The recovered geometry depicts the true geometry of the moving object, as can be seen on comparing it with figure 2 . The effect of errors in observations are also seen in the recovered geometry, when compared with true geometry shown in figure 2 .

ered geometry (figure 4) closely matches with the true geometry (figure 2). All the views of the recovered geometry including the top and 3D views are shown in figure 4 . The effect of errors in observations are visible in the recovered geometry. In figure 4 , it is visible (in both the top and side views) that the spread of points is lesser at point $H$ as compared to spread of points at points $E$ and $D$. The spread of points along the edges, which signifies the error propagated in the recovered geometry, increases as we move from point $H$ to $D$. The spread of points first decreases and then increases as we move from point $F$ to $H$. In the front view, such spread of points is not that evident though a little increase in spread of the points can be observed as one moves from point $F$ to $A$. Similarly, the spread of points first decreases and then increases as one moves from point $F$ to $H$ or from point $A$ to $B$. An immediate explanation which satisfies all of the above observations appears to be that the error at locations which are closer to point $P$ (where POS is installed, e.g. at $H$ ) is lesser as compared to locations which are farther from point $P$ (i.e. $E$ and $D)$. These observation(s) and derived explanation(s) are further verified using the results presented in the following subsections.

\subsection{Effect of system components}

This maximum error at $1 \sigma$ obtained after performing an eigen value analysis of the covariance matrix $C_{X}$ is plotted against certain parameters, the plots of which are shown in figure 5 to figure 10. The effects of parameters alone and in combination on the error of the coordinates of a point on the object are shown in generated results to study the individual and combined effects of the components. In the first case the plots of error at the said point are shown with respect to the change in laser range i.e. distance of point on the object from laser scanner (Figure 5 to Figure 7) while the distance of the point from on-board GPS is kept fixed.
In the second case the distance of point on the object from laser scanner is kept fixed and distance of the said point from on-board GPS is varied and the resulting errors are plotted (Figure 8 and Figure 9). A zoomed in view of figure 5 is shown in figure 6.

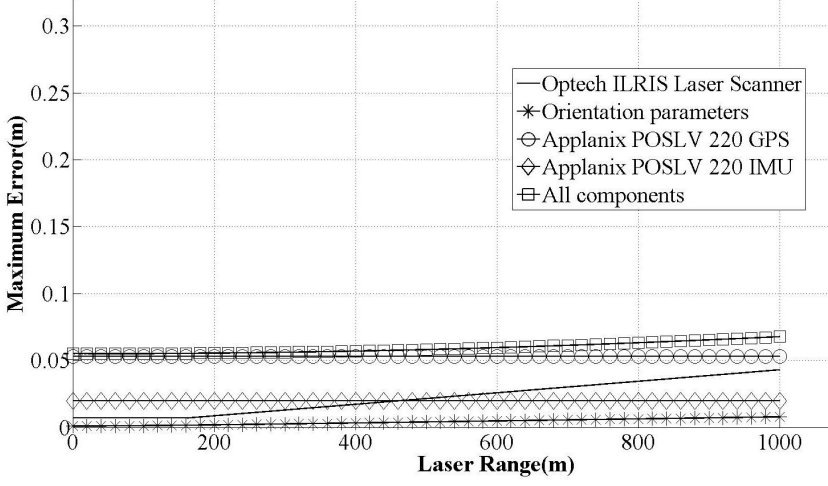

Figure 5: Effect of different components on the accuracy of computed 'motion corrected' coordinates and its variation with change in laser range. The distance of the point (on the object) from the GPS is assumed to be $40 \mathrm{~m}$.

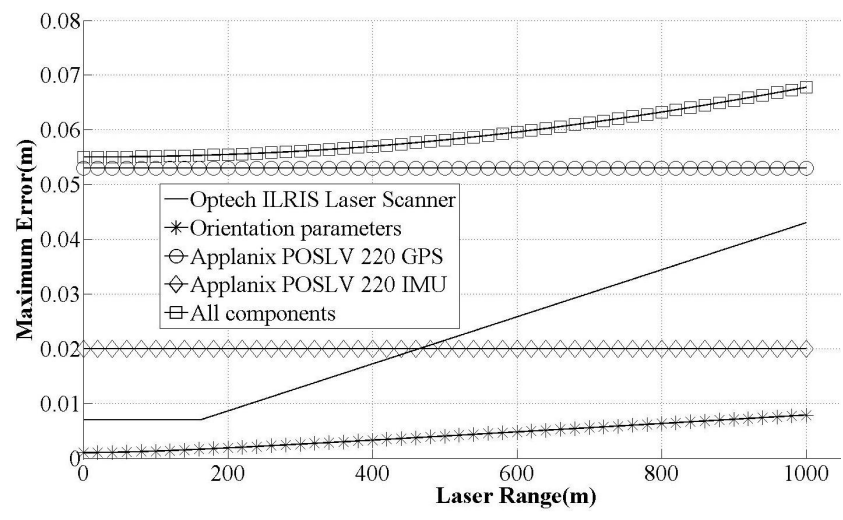

Figure 6: A zoomed in view of figure 5 showing effect of different components on the accuracy of computed 'motion corrected' coordinates and its variation with change in laser range. The distance of the point (on the object) from the GPS is assumed to be $40 \mathrm{~m}$.

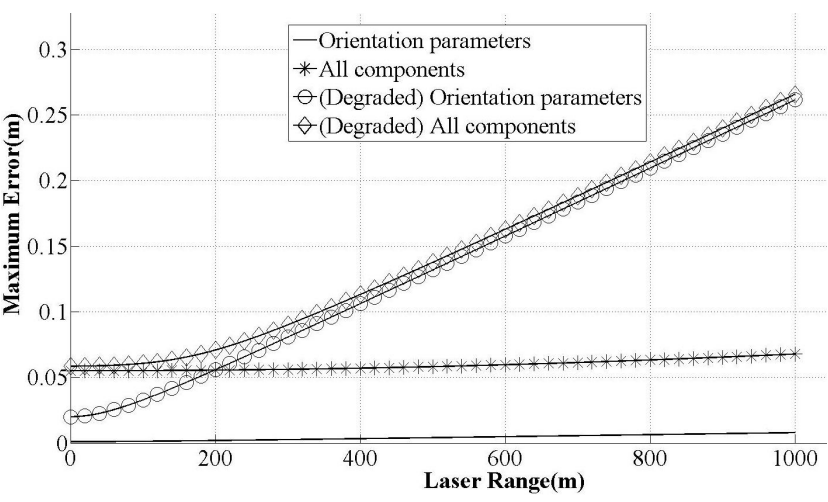

Figure 7: Effect of degrading the orientation parameters on the overall accuracy of the motion corrected coordinates. The distance of the point (on the object) from the GPS is assumed to be $40 \mathrm{~m}$.

As can be clearly seen from figure 6 , the contribution of error due to laser scanner and orientation parameters increases as the moving object goes further away. While, the effect due to GPS 
and IMU (POS system) installed on the object remains constant with increase in laser range. Since the contribution of all other error sources compared to GPS is quite insignificant, the nature of error curve is governed mostly by the GPS. When the boresight parameters are degraded as per the values given in table 3 , the contribution of all the components remain the same except for the contribution of orientation parameters which increases quite significantly. This causes a significant increase in the error due to all the components, as can be seen in figure 7 . However it is visible that at smaller ranges, the effect of orientation parameters is quite less but it increases significantly with the increase in laser range. Hence at smaller ranges, more emphasis is on GPS accuracy but for large range, accurate determination of orientation parameters particularly the angular component is quite important.

Figure 8 shows the effect of different components on the accuracy of the overall system with change in distance of point on the object from GPS while keeping the laser range to the point under consideration to be constant as $200 \mathrm{~m}$. It is visible that the effect due to orientation parameters and laser scanner is constant and does not change with change in location of POS system. This is due to the fact that errors due to laser scanner and orientation parameters depend only on the distance of the point from laser scanner and not on the distance of point from GPS, as depicted in figure 6. The effect of GPS on the system performance does not vary with the change in location of POS system. However, the contribution of error due to IMU increases as the distance of point from POS system increases. The points which are closer to the POS system are more accurate as compared to the ones which are further away. Observations similar to the above were

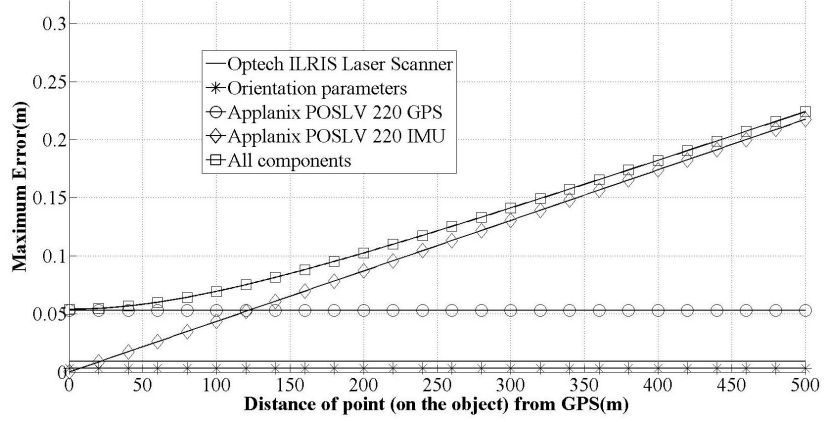

Figure 8: Effect of different components on the accuracy of computed 'motion corrected' coordinates and its variation with change in distance of the point (on the object) from GPS. The laser range is assumed to be $200 \mathrm{~m}$.

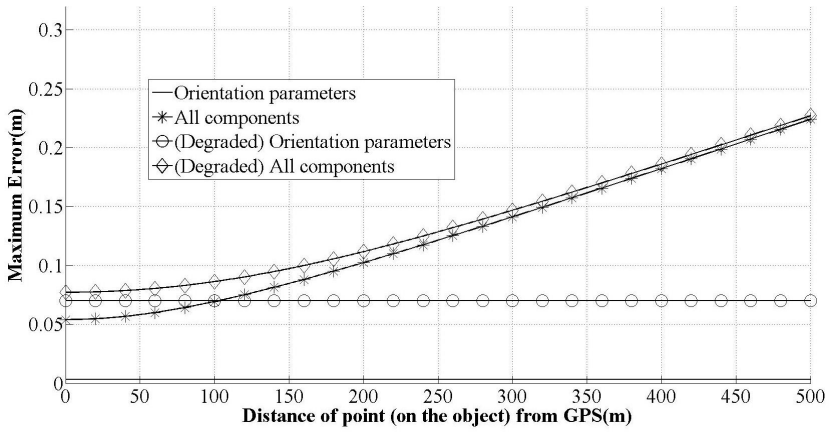

Figure 9: Effect of degrading the orientation parameters on the overall accuracy of the motion corrected coordinates. The laser range is assumed to be $200 \mathrm{~m}$.

also made in the preliminary simulation results presented in the section 4.1. In figure 4(top view), the spread of points at $D$ and
$E$ which are farthest from $P$ is more than at point $H$ which is closer to $P$. The reason for the above observation is as follows: The task performed by motion correction equation 1 is analogous to a translation to the phase center of GPS and rotation about the same point by instantaneous angles given by the IMU. Hence, the points which are further away from GPS would be more erroneous due to error in the rotational element than the points closer to the GPS. Due to the larger contribution of IMU to the error, the nature of the overall error is governed by the IMU of the POS system, as seen in figure 8 . On degrading the values of orientation parameters, the constant line shifts upwards, as can be seen in figure 9. It is interesting to note (in figure 9) that the degraded orientation parameters have a significant effect only for smaller objects. The effect of degraded orientation parameters starts to decrease as distance of GPS from the point of interest increases (i.e. as the size of object increases) and the error due to IMU start dominating. Further, as the laser range increases, the orientation parameter error curve starts shifting upwards causing the combined error curve to further shift upward. At very large range, the error due to orientation parameter would dominate. Figure 10

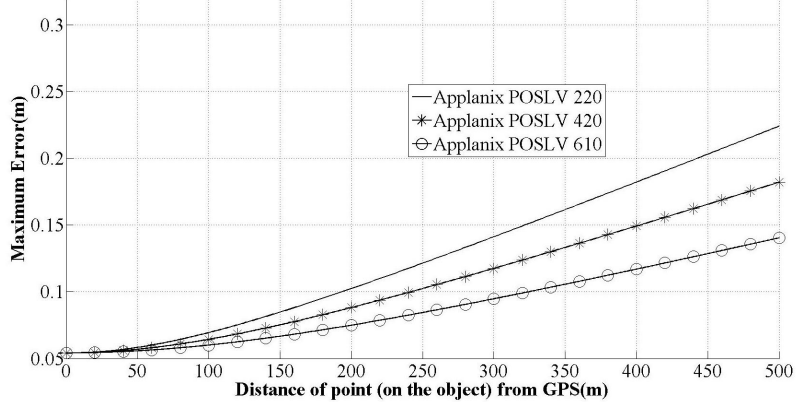

Figure 10: Performance comparison of different models of Applanix POS system for motion correction algorithm with change in distance of the point (on the object) from GPS. The distance of point from GPS is a measure of the dimensions of the object.

presents performance comparison of various POS systems offered by Applanix for their use in motion correction algorithm. The study is being carried out to see how POS system with differing accuracies and thus cost affect the motion correction performance. Also it will help to understand that for different laser ranges and different sizes of objects, how POS should be chosen intelligently to realize desired accuracy at minimal cost. These three systems perform identically for points which are closer to the POS system. Significant performance improvement for Applanix POSLV 610 is observed only for points which are farther than $250 \mathrm{~m}$ from the POS. From the observations of figure 8 and figure 10, it is evident that the GPS accuracy is crucial for points which are closer to the POS while IMU accuracy is crucial for points which are farther from the POS system. Note that the performance of POS system is independent of the laser range, as seen in figure 6.

\section{CONCLUSIONS AND FUTURE WORK}

Only three methods are available in literature which possess the capability to scan moving objects. However, the understanding of their errors and the role of various sensors and methods in motion correction process is not available. This paper has presented the motion correction algorithm by Goel and Lohani (2014) to understand the errors due to different sensor components and their effect on final coordinates using numerical simulations and verified the same using analytical understanding of the results.

As would be expected, it is found out that the accuracy of the motion corrected output decreases as the object moves away from 
the laser scanner primarily because of the presence of angular errors in the laser scanner observations. One of the most important insights obtained from this analysis is that accuracy of the obtained output not only depends on the quality of the sensors utilized but also on the location of the POS system i.e. where the POS is installed on the moving object. Intuitively, one would expect to employ a high end POS system (or IMU) for the motion correction algorithm. However it is quite clear that a high end POS system may not always be required. The quality of the POS system required depends on the dimensions of the moving object. As the dimensions of the object increase, one would want to use a POS system having a better IMU to achieve good quality results. Further, it is always advisable to install the POS system at the geometric center of the object to keep the errors minimum. For objects of small dimensions or for low range scanning projects a good quality GPS should be used, while for larger objects a better IMU is required. The effect of error in translation element of orientation parameters is more at lower ranges while the effect of angular component is visible at larger ranges. Further, the scanner orientation parameters play a more significant role for objects having smaller dimensions. As object size increases, the contribution of IMU errors starts dominating the contribution due to all other sensors.

The analysis presents important insights in to sensor selection and optimal utilization of available sensors for achieving best quality results. The future work will attempt validation of observed error patterns with experimental results and present an exhaustive sensitivity analysis of the system parameters.

\section{REFERENCES}

Blais, F., Picard, M. and Godin, G., 2004, Accurate 3D acquisition of freely moving objects, Proceedings 2nd International Symposium on 3D Data Processing, Visualization, and Transmission (3DPVT), pp. 422-429

Goel, S. and Lohani, B., 2014, A Motion Correction Technique for Laser Scanning of Moving Objects, IEEE Geoscience and Remote Sensing Letters (GRSL), 11(1), pp. 225-228

Lichti, D. D. and Gordon, S. J., 2004, Error propagation in directly georeferenced terrestrial laser scanner point clouds for cultural heritage recording, Proceedings of FIG working week, Athens, Greece.

Specifications of Optech ILRIS 3D Laser Scanner, http:// www.optech.ca/i3dprodline-ilris3d.htm (December 19, 2013).

Specifications of various POS systems offered by Applanix, http://www . applanix.com/media/downloads/products/ specs/poslv_specifications12032012.pdf (December 19, 2013).

Weise, T., Leibe, B. and Van Gool, L., 2007, Fast 3D scanning with automatic motion compensation, Proceedings IEEE Conference on Computer Vision and Pattern Recognition (CVPR). pp. $1-8$ 